\title{
Assessment of psychological pain management techniques: a comparative study between athletes and non-athletes
}

\author{
Daniel Câmara Azevedo ${ }^{1}$ and Dietmar Martin Samulski ${ }^{2}$
}

\begin{abstract}
Athletes usually deal with injuries and pain. They seem to have similar pain threshold when compared to non-athletes, although they have higher pain tolerance and the exact cause for that is unknown. High levels for pain tolerance and control can improve performance and time for injury recovery. The literature shows that use of coping strategies can increase pain control; possible differences on coping with pain between athletes and non-athletes are poorly described. The purpose of this study was to evaluate frequency of coping strategies used by athletes and nonathletes of both genders and look for possible association between preferred coping style and pain intensity. The sample included 160 subjects with actual pain experience, 80 athletes (52 male, 28 female) and 80 non-athletes (50 male and 30 female). All subjects were evaluated for pain intensity, frequency and duration and for coping strategies using a questionnaire (SBS-V). The results show that athletes and non-athletes, despite of gender, use with the same frequency coping strategies. The less common coping strategies for all groups were those poor-adaptative $(p<0.001)$; the most commonly strategy used was self-statement and regulation of body tension $(\mathrm{p}<0.001)$. Female athletes use more frequently poor-adaptative strategies when pain intensity increases $(p<0.05)$.
\end{abstract}

Key words: Pain. Athletes. Coping strategies.

1. M Sc. Assistant Professor of Physiotherapy - PUC-MG and Faculdade de Ciências Médicas de Minas Gerais.

2. $\mathrm{PhD}$. Coordinator of the Sports Psycology Laboratory - Escola de Educação Física da UFMG. Associate Professor - Escola de Educação Física da UFMG

Received in $12 / 12 / 02$

Approved in 12/7/03

Correspondence to:

Daniel Câmara Azevedo

Av. do Contorno, 9.636 - sl. 405

30110-130 - Belo Horizonte, MG

E-mail: daniel@superarfisioterapia.com.br

\section{INTRODUCTION}

About $5 \%$ to $15 \%$ of elite athletes are prevented from training for at least one month, each season, due to injuries $^{1}$. These injuries are hazardous to the athletes' health, and jeopardize their training and performance ${ }^{2}$. To live with minor or major injuries, to practice or to play under pain seem to be almost certain and acceptable experiences in the life of sportsmen and sportswomen ${ }^{3}$. Athletes seem to have pain threshold similar to non-athletes, even though their tolerance to pain is higher, the reason for this being unknown so far ${ }^{4}$.

In a number of sports, the capability to support pain can determine a higher or lower performance of an athlete, as in contact sports such as boxing ${ }^{5}$. Endurance athletes have shown that ability to tolerate high levels of pain is an important component for success in their sports modality ${ }^{6}$. According to Pen \& Fisher ${ }^{7}$, the ability of an injured athlete to support pain is related to the speed of his recovery.

Higher pain endurance capability may also directly influence training of physical abilities. Morrey ${ }^{8}$ concluded in his study that flexibility of the individuals was directly related to their ability to endure pain.

The use of psychological techniques has shown to be effective in pain control ${ }^{9-13}$. Due to the indisputable mental component of pain, such techniques, known as "coping strategies", have been broadly mentioned in the literature as effective to reduce and manage pain ${ }^{9-11}$. These techniques can be used for acute and chronic pain, and there are a number of classifications for them ${ }^{14-18}$. Haythornwaite et $a l .{ }^{18}$ defined these techniques as "an attempt to deal with or manage a specific stressor, in this case, pain, and they can be behavioral or cognitive".

The cognitive techniques typically attempt to redirect focus away from the pain or, conversely, they focus pain to reinterpret it. Other concepts stress the emotional aspects of pain, particularly anxiety and stress, on the perception of pain. In this sense, relaxing techniques are used with the purpose of decreasing the stress caused by the sensation of pain, decreasing pain and enhancing tolerance ${ }^{14}$. Behavioral techniques focus on shifting behavior in an attempt 
to manage or minimize pain. To increase or decrease the practice of a certain activity, to meet friends, to go for a walk are instances of such techniques ${ }^{10}$.

The use of these techniques is important to influence the variables of perception and endurance to pain, and they can aid the process of physical and psychological rehabilitation of athletes and non-athletes, and their performan$\mathrm{ce}^{8,19}$.

There is a great number of studies in the literature that address psychological techniques for pain control in patients with chronic and acute pain; however, there are only very few studies in populations of athletes addressing possible differences in the use of these techniques.

The study of these differences becomes relevant as one perceives differences between athletes and non-athletes in pain control and endurance. Moreover, pain in athletes is quite frequent, and its better management may promote significant performance enhancement and faster recovery from injuries.

Thus, the purposes of this study were to assess the frequency of use of pain control psychological techniques between male and female athletes and non-athletes currently experiencing pain, and to assess the relationship between the subjective perception of pain intensity and the choice of the psychological pain control technique in the four groups (male athletes, female athletes, male non-athletes, female non-athletes).

\section{METHODS}

One hundred and sixty (160) subjects took part in this study, being 80 athletes ( 52 males and 28 females) and 80 non-athletes (50 males and 30 females). Athletes for the study were those subjects who practiced sports regularly seeking to enhance their performance and who took part in amateur and/or professional competitions regularly. Nonathletes were those who did not practice sports regularly (no more than once a week). All subjects were undergoing physical therapy rehabilitation and said they were currently experiencing pain.

The subjects were divided in four groups: MA - male athletes $(\mathrm{n}=52)$, FA - female athletes $(\mathrm{n}=28)$, MNA male non-athletes $(n=50)$, and FNA - female non-athletes $(\mathrm{n}=30)$.

As the subjects of the sample were currently experiencing pain and undergoing physical therapy rehabilitation, data collection was made in physical therapy clinics (SPORTS - Centro de Reabilitação, IOT-BH, NOT, FISIOR), medical departments of sports clubs (Minas Tênis Clube, Clube Atlético Mineiro), and the Prevention and Rehabilitation of Sports Injuries Lab, (LAPREV), from the Minas Gerais Federal University (UFMG) Center of Excellence in
Sports. Prior to data collection, the purposes and procedures related to the investigation were explained to those in charge of these institutions, who gave their consent.

In addition, an informed consent form stating the purposes, data collection method, possible risks and benefits related to the study was attached to the data collection form. After reading it, this form should be signed by the subject, agreeing to take part in the study. This study was approved by UFMG Research Ethics Committee.

Data collection was made with the use of a tool (questionnaire) with two parts. In the first part, the subject should indicate his/her age, gender, and provide information on the practice of sports: whether or non-athlete, and mean intensity of pain. For this measure, a visual scale of the subjective perception of pain ${ }^{20}$ was used, and the subject had to rate the intensity of pain out of 10 possible scores.

The use of scales to measure pain intensity has become common in pain-related studies, showing good reliabili$\operatorname{ty}^{21-24}$.

In the second part of the tool, the frequency of use of psychological techniques for pain control was assessed through a SBS-V questionnaire validated by Kleinert ${ }^{15}$. This tool was originally designed in German, and was validated for athletes and non-athletes with chronic and acute pain. This tool was translated into Portuguese and then translated back to German, by different professionals well versed in both languages. After re-translation to German, it was compared to the original version to confirm their similarity.

In this questionnaire, a list of 27 pain control psychological techniques is presented. For each technique, the subject should inform how often he used it when feeling pain. There were six possible scores, being "1" related to "always" and " 6 " to "never".

The SBS-V questionnaire is based on the eight dimensions pain control psychological techniques classification, being the first one considered poor-adaptative, and the other seven, adaptative techniques. Higher use of adaptative techniques are related to the improvement of pain-related medical parameters, whereas higher use of poor-adaptative techniques are related to the worsening of the pain-related medical parameters ${ }^{25}$. The eight dimensions are:

1) Poor-adaptative techniques - They indicate an improper regulation of the pain control process. It is observed in resignation and denial behavior.

2) Motivational techniques - This implies self-instructions of motivational character and positive self-instructions to control pain.

3) Body tension regulation - Through relaxation and change of posture the muscle tension level will be regulated, in an attempt to control pain. 
TABLE 1

Descriptive and comparative analysis of the subjects' age among the four groups

\begin{tabular}{cccccccc}
\hline \multirow{2}{*}{ Variable } & Group & \multicolumn{5}{c}{ Descriptive measures } & P \\
\cline { 3 - 6 } & & Minimum & Maximum & Median & Mean & s.d. \\
& & & & & & \\
Age (years) & AF & 18.0 & 31.0 & 25.0 & 23.9 & 4.2 & 0.268 \\
& NAM & 18.0 & 36.0 & 23.5 & 24.5 & 5.2 & \\
& NAF & 18.0 & 35.0 & 25.0 & 25.6 & 4.5 \\
& 18.0 & 33.0 & 22.5 & 24.4 & 4.9 \\
\hline
\end{tabular}

Note: The value of $\mathbf{p}$ in the table relates to the probability of Kruskal-Wallis test being significant.

Legend: GROUP $\rightarrow$ AM - Male athlete; AF - Female athlete; NAM - Male non-athlete; NAF - Female non-athlete.

4) Search for information - It implies the assessment of pain and search for information on the pain phenomenon.

5) Body activity - Through motion, one tries to shift the focus away from pain or to relieve it.

6) Setting of goals - This is shown in the understanding and planning of goals for pain control.

7) Comparison techniques - This implies the use of painrelated intra- or interpersonal comparisons.

8) Distractive techniques - The use of different forms of distraction from pain, such as social activities, music, TV, video.

Two techniques were not used in the comparison among dimensions as they did not fit among the different dimensions, according to a previous assessment of factors.

Data collection was carried out in physical therapy clinics, medical department of the sports clubs previously mentioned, and the CENESP-UFMG Sports Injuries Prevention and Rehab Lab. The patients were approached before starting physical therapy, and were informed on the study by the investigator in charge of it. After signing the informed consent form, they received the instructions for filling out the questionnaire, which should be done without intervention from the investigator.

All subjects should, in an interview with the investigator before filling out the questionnaire, state that they were currently experiencing pain, and meet the criteria of athlete and non-athlete established in the investigation.

For the statistical analysis of the collected data, KruskalWallis' test, Friedman's test, and Pearson's correlation analysis were used.

All results were considered significant, on a significance level of $5 \%(p<0.05)$, therefore, with a $95 \%$ of confidence that the results are correct.

\section{RESULTS}

A total of 160 subjects were interviewed, being 80 athletes (52 males and 28 females), and 80 non-athletes (50
TABLE 2

Descriptive and comparative analysis of the measure that assess mean intensity of pain among the four groups

\begin{tabular}{cccccc}
\hline \multirow{2}{*}{ Variable } & Group & \multicolumn{3}{c}{ Descriptive measures } & p \\
\cline { 3 - 4 } & & Minimum & Maximum & Median & \\
& & & & & \\
Intensidade & AF & 2.0 & 9.0 & 5.0 & 0.131 \\
da dor & NAM & 2.0 & 8.0 & 5.5 & \\
& NAF & 2.0 & 8.0 & 4.0 & \\
& 2.0 & 9.0 & 5.0 & \\
\hline
\end{tabular}

Note: The value of $\mathbf{p}$ in the table relates to the probability of Kruskal-Wallis test being significant.

Legend: GROUP $\rightarrow$ AM - Male athlete; AF - Female athlete; NAM - Male non-athlete; NAF - Female non-athlete.

males and 30 females). Table 1 shows there was no significant difference $(p>0.05)$ among the four groups of interviewees according to age, i.e, the four groups had an homogeneous distribution in regard to this variable.

No significant difference was observed $(\mathrm{p}>0.05)$ among the four groups of interviwees in terms of the mean intensity of pain, i.e., the four groups of interviwees had similar measurements on pain assessment. This result can be seen in table 2 .

Tables 3 and 4 showed a comparison among the four groups in relation to the frequency each pain control psychological technique was used.

In relation to the frequency of use of poor-adaptative techniques (dimension I), the results in the four groups showed high values (ranging from 4.8 to 5.0), evidencing low frequency of use of these techniques $(1=$ always use, $6=$ never use). Comparison of results among the four groups shows there was no significant differences $(p>0.05)$ among them, i.e., the four groups use these techniques with the same frequency.

Motivational techniques (dimension II) scored low among all groups (between 2.1 and 2.2), showing they are 
highly used by the four groups, with no significant differences among them. Similar results were observed in the techniques of body tension regulation (dimension III). In this dimension, again, all groups presented low scores (ranging from 1.8 to 2.7 ) for all groups, meaning that them all often use these techniques in an attempt to control pain, again with no significant differences among the groups.

The techniques related to searching for information (dimension IV) were also highly used by all groups (2.3 to 3.0 ), even though their mean values were a little higher (less frequency of use) than those for dimensions II and III. Again there were no significant differences in this dimension among the four groups.

Body activity (dimension V) had medium values for all groups, evidencing a medium frequency of use. Even though the values showed group AF (value $=3.2$ ) to use this technique the most when compared to groups AM (val-

TABLE 3

Descriptive and comparative analysis among the four groups investigated in relation to the frequency of use of dimensions I, II, III, IV and V

\begin{tabular}{|c|c|c|c|c|c|}
\hline \multirow[t]{2}{*}{ Dimensions } & \multirow[t]{2}{*}{ Group } & \multicolumn{3}{|c|}{ Descriptive measures } & \multirow[t]{2}{*}{$\mathbf{P}$} \\
\hline & & Minimum & Maximum & Median & \\
\hline \multirow[t]{4}{*}{1} & $A M$ & 2.4 & 6.0 & 4.8 & \multirow[t]{4}{*}{0.127} \\
\hline & $\mathrm{AF}$ & 3.6 & 6.0 & 4.8 & \\
\hline & NAM & 2.2 & 6.0 & 5.0 & \\
\hline & NAF & 3.2 & 6.0 & 4.8 & \\
\hline \multirow[t]{4}{*}{ ॥ } & AM & 1.0 & 4.7 & 2.1 & \multirow[t]{4}{*}{0.579} \\
\hline & $\mathrm{AF}$ & 1.0 & 4.7 & 2.2 & \\
\hline & NAM & 1.0 & 4.7 & 2.2 & \\
\hline & NAF & 1.0 & 4.7 & 2.2 & \\
\hline \multirow[t]{4}{*}{ III } & $A M$ & 1.0 & 4.7 & 2.0 & \multirow[t]{4}{*}{0.337} \\
\hline & $\mathrm{AF}$ & 1.0 & 3.3 & 1.8 & \\
\hline & NAM & 1.0 & 5.0 & 2.0 & \\
\hline & NAF & 1.3 & 4.3 & 2.7 & \\
\hline \multirow[t]{4}{*}{ IV } & AM & 1.0 & 5.3 & 2.3 & \multirow[t]{4}{*}{0.082} \\
\hline & $\mathrm{AF}$ & 1.0 & 5.3 & 3.0 & \\
\hline & NAM & 1.0 & 5.3 & 3.0 & \\
\hline & NAF & 1.0 & 5.3 & 2.3 & \\
\hline \multirow[t]{4}{*}{ V } & $A M$ & 1.0 & 6.0 & 3.5 & \multirow[t]{4}{*}{0.069} \\
\hline & $\mathrm{AF}$ & 1.0 & 6.0 & 3.2 & \\
\hline & NAM & 1.0 & 6.0 & 4.0 & \\
\hline & NAF & 1.0 & 6.0 & 4.0 & \\
\hline
\end{tabular}

Note: The value of $\mathbf{p}$ in the table relates to the probability of Kruskal-Wallis test being significant.

Legend: GROUPS $\rightarrow$ AM - Male athlete; AF - Female athlete; NAM - Male non-athlete; NAF - Female non-athlete.

DIMENSIONS $\rightarrow \mathbf{I}$ - Poor-adaptative techniques; II - Motivational techniques; III - Body tension regulation; IV - Search for information; $\mathbf{V}$ - Body activity.

SCORES $\rightarrow \mathbf{1}=$ Always use; $\mathbf{6}=$ Never use. ue $=3.5)$, NAM (value $=4.0)$ and NAF (value $=3.9)$, this difference was not considered statistically significant $(\mathrm{p}=$ 0.069).

Techniques for setting of goals (dimension VI) also present high frequency of use (values between 2.3 and 2.7), with no significant differences observed among the groups.

Comparison techniques (dimension VII) and body activity techniques presented medium frequency of use, with values between 3.5 and 4.0, with no significant differences among the four groups.

Distractive techniques (dimension VIII) stand out for the high values found in all groups (between 4.3 and 4.5). Thus, these techniques, in spite of being considered adaptative, had low frequency of use among all groups, with no significant differences among them.

Accordingly, the presented results show there were no significant differences when comparing the frequency of use of each psychological technique dimension among the groups.

When assessing the frequency of use of the different dimensions of pain control psychological techniques in each group, there were significant differences $(p<0.05)$ in the four groups investigated (tables 5 and 6 ).

In group AM, techniques most significantly ( $p<0.001)$ used were motivational techniques, body tension regulation, search for information and setting of goals, with no

\begin{tabular}{|c|c|c|c|c|c|}
\hline \multicolumn{6}{|c|}{$\begin{array}{c}\text { TABLE } 4 \\
\text { Descriptive and comparative analysis among the four } \\
\text { groups investigated in relation to the frequency } \\
\text { of use of dimensions VI, VII and VIII }\end{array}$} \\
\hline \multirow[t]{2}{*}{ Dimensions } & \multirow[t]{2}{*}{ Group } & \multicolumn{3}{|c|}{ Discriptive measures } & \multirow[t]{2}{*}{$\mathbf{P}$} \\
\hline & & Minimum & Maximum & Median & \\
\hline \multirow[t]{4}{*}{$\mathrm{Vl}$} & AM & 1.0 & 4.7 & 2.3 & \multirow[t]{4}{*}{0.723} \\
\hline & $\mathrm{AF}$ & 1.0 & 5.3 & 2.7 & \\
\hline & NAM & 1.0 & 5.7 & 2.7 & \\
\hline & NAF & 1.0 & 5.7 & 2.7 & \\
\hline \multirow[t]{4}{*}{ VII } & $A M$ & 1.0 & 6.0 & 3.5 & \multirow[t]{4}{*}{0.728} \\
\hline & $\mathrm{AF}$ & 1.0 & 6.0 & 3.5 & \\
\hline & NAM & 1.0 & 6.0 & 4.0 & \\
\hline & NAF & 1.0 & 6.0 & 3.7 & \\
\hline \multirow[t]{4}{*}{ VIII } & AM & 1.3 & 6.0 & 4.3 & \multirow[t]{4}{*}{0.181} \\
\hline & $\mathrm{AF}$ & 2.0 & 6.0 & 4.5 & \\
\hline & NAM & 3.0 & 6.0 & 4.3 & \\
\hline & NAF & 1.0 & 6.0 & 4.3 & \\
\hline
\end{tabular}

Note: The value of $\mathbf{p}$ in the table relates to the probability of Kruskal-Wallis test being significant.

Legend: GROUPS $\rightarrow$ AM - Male athlete; AF - Female athlete; NAM - Male non-athlete NAF - Female non-athlete.

DIMENSIONS $\rightarrow \mathbf{V I}$ - Setting of goals; VII - Comparison techniques; VIII - Distractive techniques.

SCORES $\rightarrow \mathbf{1}=$ Always use; $\mathbf{6}=$ Never use 
significant differences among them. Next, body activity and comparison techniques had medium frequency of use (value $=3.5)$, with significant differences in relation to other techniques $(p<0.001)$. Distractive techniques $(p<0.001)$ were, among the adaptative techniques, the least used (value $=4.3$ ), even though their frequency of use was higher ( $p$ $<0.001$ ) when compared to poor-adaptative techniques (value $=4.8$ ).

In group AF, the techniques most often used (values from 1.8 to 2.2 ) were motivational techniques and body tension regulation $(\mathrm{p}<0.001)$. Also with high frequency (values between 2.7 and 3.5), but significantly lower ( $<0.001)$, are search for information, body activity, setting of goals, and comparison techniques. For this group, poor-adapta-

\begin{tabular}{|c|c|c|c|c|c|}
\hline & $\begin{array}{l}\text { Descripti } \\
\text { among the } \\
\text { dimensions }\end{array}$ & $\begin{array}{r}\text { TABL } \\
\text { ve and con } \\
\text { frequencie } \\
5 \text { for group }\end{array}$ & $\begin{array}{l}\text { E } 5 \\
\text { iparative ar } \\
\text { s of use of } \\
\text { S AM, AF a }\end{array}$ & $\begin{array}{l}\text { nalysis } \\
\text { the eight } \\
\text { nd NAM }\end{array}$ & \\
\hline Group & Dimensions & Descr & ptive meast & ures & $\mathbf{P}$ \\
\hline & & Minimum & Maximum & Median & \\
\hline$A M$ & I & 2.4 & 6.0 & 4.8 & $<0.001$ \\
\hline & II & 1.0 & 4.7 & 2.1 & \\
\hline & III & 1.0 & 4.7 & 2.0 & \\
\hline & IV & 1.0 & 5.3 & 2.3 & \\
\hline & V & 1.0 & 6.0 & 3.5 & \\
\hline & $\mathrm{VI}$ & 1.0 & 4.7 & 2.3 & \\
\hline & VII & 1.0 & 6.0 & 3.5 & \\
\hline & VIII & 1.0 & 6.0 & 4.3 & \\
\hline$A F$ & I & 3.6 & 6.0 & 4.8 & $<0.001$ \\
\hline & II & 1.0 & 4.7 & 2.2 & \\
\hline & III & 1.0 & 3.3 & 1.8 & \\
\hline & IV & 1.0 & 5.3 & 3.0 & \\
\hline & V & 1.0 & 6.0 & 3.2 & \\
\hline & $\mathrm{VI}$ & 1.0 & 5.3 & 2.7 & \\
\hline & VII & 1.0 & 6.0 & 3.5 & \\
\hline & VIII & 2.0 & 6.0 & 4.5 & \\
\hline NAM & I & 2.4 & 6.0 & 5.0 & $<0.001$ \\
\hline & II & 1.0 & 4.7 & 2.2 & \\
\hline & III & 1.0 & 5.0 & 2.0 & \\
\hline & IV & 1.0 & 5.3 & 3.0 & \\
\hline & V & 1.0 & 6.0 & 4.0 & \\
\hline & VI & 1.0 & 5.7 & 2.7 & \\
\hline & VII & 1.0 & 6.0 & 4.0 & \\
\hline & VIII & 3.0 & 6.0 & 4.3 & \\
\hline
\end{tabular}

Note: The value of $\mathbf{p}$ in the table relates to the probability of Friedman's test being significant.

Legenda: GROUPS $\rightarrow$ AM - Male athlete; AF - Female athlete; NAM - Male non-athlete.

DIMENSIONS $\rightarrow$ I- Poor-adaptative techniques; II - Motivational techniques; III - Body tension regulation; IV - Search for information; $\mathbf{V}$ - Body activity; VI - Setting of goals; VII - Comparison techniques; VIII - Distractive techniques.

SCORES $\rightarrow \mathbf{1}=$ Always use; $\mathbf{6}=$ Never use. tive techniques and distractive techniques were the least frequent $(\mathrm{p}<0.001)$, but with no significant differences among them (values $=4.8$ and 4.5 respectively).

Group NAM and group AF evidenced body tension regulation (value $=2.0$ ) and motivational techniques (value $=$ $2,2)$ as the most used $(\mathrm{p}<0.001)$. Still with high, but lower frequency $(\mathrm{p}<0.001)$, there are search for information (value $=3.0$ ) and setting of goals techniques (value $=2.7$ ), with no significant differences among them. Comparison techniques and body activity present medium frequency of use for pain control (value $=4.0$ ), and their frequency of use is higher $(\mathrm{p}<0.001)$ when compared to distractive techniques (value $=4.3$ ) and poor-adaptative techniques (value $=5.0$ ), with statistically similar frequency.

For group NAF, the most used techniques $(\mathrm{p}<0.001)$ were motivational techniques (value $=2.2$ ), body tension regulation $($ value $=2.7)$, search for information (value $=$ 2.3 ) and setting of goals (value $=2.7$ ), with no significant differences among them. With a lower frequency of use (values from 3.7 to 4.3 ), are the comparison techniques, body activity and distractive techniques $(\mathrm{p}<0.001)$. For this group, poor-adaptative techniques (value $=4.8$ ) were, alone, the least frequently used $(\mathrm{p}<0.001)$.

In short, among all groups, poor-adaptative techniques were, separately or not, the techniques used the least. Among the adaptative techniques, the distractive techniques were the least used. Motivational techniques and body tension regulation were, separately or not, the most used techniques. The results described here can be better assessed in tables 5 and 6.

\section{TABLE 6}

Descriptive and comparative analysis among the frequencies of use of the eight dimensions for group NAF

\begin{tabular}{|c|c|c|c|c|c|}
\hline \multirow[t]{2}{*}{ Group } & \multirow[t]{2}{*}{ Dimensions } & \multicolumn{3}{|c|}{ Descriptive measures } & \multirow[t]{2}{*}{$\mathbf{P}$} \\
\hline & & Minimum & Maximum & Median & \\
\hline \multirow[t]{8}{*}{ NAF } & I & 3.2 & 6.0 & 4.8 & $<0.001$ \\
\hline & II & 1.0 & 4.7 & 2.2 & \\
\hline & III & 1.3 & 4.3 & 2.7 & \\
\hline & IV & 1.0 & 5.3 & 2.3 & \\
\hline & V & 1.0 & 6.0 & 4.0 & \\
\hline & VI & 1.0 & 5.7 & 2.7 & \\
\hline & VII & 1.0 & 6.0 & 3.7 & \\
\hline & VIII & 1.0 & 6.0 & 4.3 & \\
\hline
\end{tabular}

Note: The value of $\mathbf{p}$ in the table relates to the probability of Friedman's test being significant.

Legend: GROUPS $\rightarrow$ NAF - Female non-athlete.

DIMENSIONS $\rightarrow$ I - Poor-adaptative techniques; II - Motivational techniques; III - Body tension regulation; IV - Search for information; V - Body activity; VI - Setting of goals; VII - Comparison techniques; VIII - Distractive techniques.

SCORES $\rightarrow \mathbf{1}=$ Always use; $\mathbf{6}=$ Never use 
TABLE 7

Correlation analysis between the measure that evaluates pain intensity and frequency of use of each one of the eight dimensions assessed, per group

\begin{tabular}{|c|c|c|c|c|c|c|c|c|}
\hline \multirow[t]{3}{*}{ Dimension } & \multicolumn{8}{|c|}{ Group } \\
\hline & \multicolumn{2}{|c|}{ AM } & \multicolumn{2}{|c|}{ AF } & \multicolumn{2}{|c|}{ NAM } & \multicolumn{2}{|c|}{ NAF } \\
\hline & $\mathbf{R}$ & $\mathbf{P}$ & $\mathbf{R}$ & $\mathbf{P}$ & $\mathbf{R}$ & $\mathbf{P}$ & $\mathbf{R}$ & $\mathbf{P}$ \\
\hline I & -0.14 & 0.337 & -0.47 & 0.012 & -0.16 & 0.279 & 0.13 & 0.490 \\
\hline ॥ & -0.14 & 0.330 & 0.02 & 0.904 & 0.16 & 0.257 & 0.32 & 0.090 \\
\hline III & 0.04 & 0.760 & -0.06 & 0.745 & 0.14 & 0.330 & -0.16 & 0.390 \\
\hline IV & 0.16 & 0.257 & -0.03 & 0.891 & 0.07 & 0.643 & -0.24 & 0.216 \\
\hline V & 0.23 & 0.104 & -0.04 & 0.844 & 0.17 & 0.234 & -0.04 & 0.817 \\
\hline $\mathrm{VI}$ & 0.02 & 0.871 & 0.12 & 0.551 & 0.08 & 0.584 & -0.09 & 0.638 \\
\hline VII & -0.15 & 0.296 & 0.18 & 0.363 & -0.14 & 0.354 & -0.25 & 0.188 \\
\hline VIII & -0.08 & 0.558 & -0.04 & 0.838 & -0.19 & 0.181 & -0.27 & 0.150 \\
\hline
\end{tabular}

Note: The value of $\mathbf{p}$ in the table relates to the probability of Pearson's correlation analysis being significant. - $\mathbf{r}$ - correlation coefficient.

Legend: GROUPS $\rightarrow$ AM - Male athlete; AF - Female athlete; NAM - Male non-athlete; NAF - Female non-athlete. DIMENSIONS $\rightarrow \mathbf{I}$ - Poor-adaptative techniques; II - Motivational techniques; III - Body tension regulation; IV Search for information; V - Body activity; VI - Setting of goals; VII - Comparison techniques; VIII - Distractive techniques.

Table 7 shows that, for group AF, there was a negative correlation of moderate value between the mean perception of pain intensity and the use of poor-adaptative techniques. This means that as the average intensity of pain increases, also the frequency of use of poor-adaptative techniques increases $(\mathrm{p}<0.05)$. There were no correlations between average perception of pain intensity and poor-adaptative techniques for the other groups, and of the other dimensions assessed for the four groups investigated.

\section{DISCUSSION}

This study did not demonstrate significant difference among athletes and non-athletes of both genders in relation to the frequency of use of psychological techniques for pain control. The previously suggested hypothesis that athletes would use adaptative techniques the most, or that athletes would use less the poor-adaptative techniques, was ruled out. We do not know of other studies that compared these populations. The possible explanation for athletes to better endure pain ${ }^{4,5}$ due to higher use of pain control psychological techniques seems unlikely.

The exact cause for this higher endurance capability remains unknown, even though there are some theories to explain it, among them the higher experience with pain the athlete has would provide him/her with a more realistic meaning of what pain actually is, thus making him/her less fearful of it; or the athlete would develop physiological features that would enable higher pain tolerance and control ${ }^{5}$.
The purpose of this investigation was to study the frequency of use of the different psychological techniques for pain control; therefore, it was not possible to assess other variables that may influence pain tolerance and control, such as when and how these techniques were employed ${ }^{26}$. According to Keefe et al. ${ }^{26}$ and Syrjala et al. ${ }^{11}$, in addition to use less the poor-adaptative techniques or to use more the adaptative techniques, the individual should learn how and when to use these techniques; thus, some techniques would be more effective in particular situations. A right method to use these techniques, based on a systematic learning and training program, would be ideal before using psychological techniques for pain control.

Based on similar frequencies found between athletes and non-athletes, we can imagine that the use by athletes of psychological techniques for pain control is intuitive, and not as consequence of any training or methodology. This idea reinforces the need for a training methodology of these techniques as an important component of the process of the athlete's shaping and training. As the athlete is often in pain, the question is: if athletes used psychological pain control measures more often, would they have higher endurance to pain compared to non-athletes? Or: would their performance enhance, as some studies have shown that the use of these techniques allows for the enhancement of physical performance ${ }^{8,15}$ ? Moreover, a higher control over pain is related, many a time, with a swifter recovery from injuries $^{7}$, being the use of these techniques recommended for both populations (athletes and non-athletes). 
We could observe that poor-adaptative techniques were those used the least in all groups $(\mathrm{p}<0.001)$. This type of technique, when used often, is an inadequate attempt to regulate pain; therefore, it is ideal to find values close to 6 ("I never use"). Even though its values for the four groups evidences low frequency of use (between 4.8 and 5.0), the question remains on the possible effect of a systematic training to increase these values (i.e., to decrease frequency).

As to the mean intensity of pain, the average of the values found were similar for the four groups. The scored value could range from 0 (no pain sensation) to 9 (the most extreme pain sensation imaginable), and the values of the groups always ranged between 4.0 (male non-athlete) and 5.5 (female athlete), which characterizes average intensity pain. These data are similar to the ones from the study carried out by Kleinert \& Samulski ${ }^{20}$ in which the average pain in a population of young athletes was of 4.9.

The hypothesis previously raised on the possible correlation between pain intensity and the selection of a specific dimension or pain control adaptative technique was demonstrated in our investigation. However, the poor-adaptative techniques $(\mathrm{p}<0.05)$ were only for group AF. This is a moderate negative correlation $(r=-0.47)$, i.e., as the intensity of pain increases, group AF uses poor-adaptative techniques the more (score $1=$ always use, score $6=$ never use). This correlation shows a tendency of this group to adopt improper pain control measures for higher intensities of pain. Other authors ${ }^{27-30}$ have found similar correlations, even though all of them assessed an ordinary population with chronic pain, which makes comparisons difficult. The exact reason the correlation between these variables occurred only for this group is unknown. In the other groups investigated, the choice of a poor-adaptative technique seems to be consequence of a personal assessment, regardless of the intensity of the perceived pain. The fact of being athlete and female did not determine, in this study, a higher frequency of use of poor-adaptative techniques, even though the literature advocates a higher tendency of women using poor-adaptative techniques ${ }^{31}$.

A number of studies are being made with the purpose of exploring possible differences between genders in relation to the use of psychological techniques for pain control ${ }^{20,29}$, 31,32 . This study failed to demonstrate possible differences between genders in relation to the frequency of use of the different psychological techniques dimensions for pain control. In both populations, of athletes and non-athletes, there were no significant differences between genders. These results confirm the findings of Kolt et al. ${ }^{32}$. They studied a population of athletes, compared males and females and did not find significant differences between genders, except for a dimension called "seek for social sup- port", which is more used by women than by men. Kleinert \& Samulski ${ }^{20}$ observed that women used psychological techniques for pain control more often, which goes against the results of our study. A possible explanation for this difference is the use of different data collection instruments. A hurdle for comparing the results from different studies is the existence of a number of tools to measure frequency of use of psychological techniques for pain control available in the literature ${ }^{25,33,34}$, in addition to the different classifications of these techniques. The existence of a number of tools and classifications makes comparisons among studies difficult, as apparently similar techniques present, many a time, conceptual differences. A same dissociative technique may be called differently in different studies, such as focused attention technique or somatization technique ${ }^{10}$. Thus, for higher exploration of the effectiveness and features of psychological techniques for pain control, the terminology and the instruments should be unified, to enable comparative studies.

According to Reid et al. mentioned by Unruh ${ }^{31}$, women are more likely to use catastrophization (poor-adaptative technique) when compared to men. Unruh, reviewing the subject $^{31}$, observed that women have typically higher repertoire of pain control techniques, including active behavior, cognitive techniques, avoidable techniques, emotional-focused strategies, seeking for social support, relaxation, and distraction, whereas men have a more direct behavior on possible actions, problem-focused strategies, denial, redefinition, activities to reduce tension (use of alcohol, tobacco and drugs). It is to be mentioned that the samples used in these studies are of patients with pain of long duration, and this is a possible explanation for differences in relation to this study, as in our sample only $39.15 \%$ of the subjects had pain for more than six months (chronic pain).

In terms of pain control adaptative techniques, one can see that distractive techniques were, among the adaptative techniques, those used the least by all groups. This type of technique is based on shifting the focus away from pain, and its effectiveness in reducing the perception of pain in cases of acute and chronic pain is very clear in the literature ${ }^{12,13,35,36}$. The low frequency of use of this technique supports the idea that the utilization of psychological techniques for pain control in athletes and non-athletes is based on intuiton, and not in previous information on the effectiveness and methodology for their use.

Motivational techniques (cognitive) and body tension regulation (behavioral) were the techniques used the most by all groups. These data confirm the findings of Kleinert $\&$ Samulski ${ }^{20}$, who observed that young athletes $(<21$ years old) had the highest frequency of use of body regulation techniques. The use of this technique would be related to a 
number of positive effects, some of them related to pain. Among them, decrease of heart rate, decrease of respiration rate, increase of oxygen uptake, decrease of muscle tension, decrease of the galvanic response of the skin, redistribution of the blood flow, decrease of blood pressure, decrease of lactate and cholesterol, increase of stress reactivity, enhancement of the immune system, and decrease of cortisol that inhibits reparation os muscle fibers ${ }^{38}$. According to Haythornwaite et al. ${ }^{18}$, the use of motivational techniques (called self-motivation by the author) would be related to a higher perception of pain control.

Setting of goals and search for information had an overall high frequency for all groups, being more used by groups AM and NAF. According to Ievleva \& Orlick ${ }^{39}$, the highest use of the setting of goals techniques is related to a swifter recovery from injuries.

In general, we could see that, for all groups, the distribution of the frequency of use of psychological techniques for pain control present little variation, being worthy of mention the high frequency of utilization of motivational techniques and body tension regulation, and the low frequency of use of poor-adaptative techniques, similar to the frequency of use of distractive techniques for groups $\mathrm{AF}$ and NAM.

\section{CONCLUSIONS AND RECOMMENDATIONS}

Athletes and non-athletes of both genders use psychological adaptative and poor-adaptative pain control techniques with the same frequency. Female athletes use significantly more $(p<0.05)$ the poor-adaptative psychological techniques as the average intensity of pain increases. Pooradaptative psychological techniques, followed by distractive techniques, are the least often used by all four groups investigated ( $p<0.001)$. Motivational techniques and body tension regulation are the techniques most often used by all four groups $(\mathrm{p}<0.001)$.

The purpose of this investigation was to assess possible differences in the frequency of use of psychological techniques for pain control in athletes and non-athletes of both genders. The literature shows that, in addition to using these techniques more or less often, the assessment of when and how individuals use them is necessary for a better understanding of the influence of these techniques on pain control. Studies assessing these factors could, perhaps, elucidate the differences among these populations.

The high number of tools and classifications of psychological techniques for pain control jeopardize the comparison of the different results presented in the literature. Unifying classifications and tools is, perhaps, an important step to achieve further conclusions on this theme.
Through the review of the literature we carried out, one can see that the study of psychological techniques for pain control is still very incipient in the field of sports, which makes comparisons with other studies on the subject more difficult, a fact that does not happen with studies investigation populations of patients with chronic pain. We hope this study may encourage further ones, as these techniques seem to significantly influence pain control, the speed of injury recovery, and the performance of athletes.

The effectiveness of the psychological intervention on pain points out the need for one to investigate the effects of combined programs of different disciplines (psychology, physical therapy, medicine) on recovery of injuries.

The similar data found in comparing the frequency of use of psychological techniques between athletes and nonathletes reinforce the idea that this type of techniques is not present or systematically used in sports. Therefore, the need to disseminate this type of techniques among athletes, coaches, physical trainers, physicians, physical therapists, psychologists, among other sports professionals, is clear.

Longitudinal studies investigating the progression of the frequency of use of these techniques after a systematic training would be interesting, as they would allow for a more appropriate assessment of the influence of these techniques on parameters for pain (intensity), incidence of injuries, and perception of pain control.

All the authors declared there is not any potential conflict of interests regarding this article.

\section{REFERENCES}

1. Engstrom R, Johansson M, Tornkvist M, Forssblad M. Does a major knee injury definitely sideline an elite soccer player? Am J Sports Med 1990;18:101-5.

2. Bajin B. An analysis of injuries in amateur sport, in particular women's gymnastics. Unpublished observations. Otawa: Canadian Gymnastic Federation, 1982.

3. Gauron EF, Bowers WA. Pain control techniques in college-age athletes. Psychol Rep 1986;59:1163-8.

4. Yaffe M. Sports injuries - Psychological aspects. British Journal of Hospital Medicine 1983;224-32.

5. Ryan ED, Kovacic CR. Pain tolerance and sports participation. Percept Mot Skills 1966;22:383-90.

6. Egan S. Acute-pain tolerance among athletes. Canadian Journal of Sport Sciences $1987 ; 12: 175-8$

7. Pen LJ, Fisher CA. Athletes and pain tolerance. Sports Med 1994;18: 319-29.

8. Morrey MA. A longitudinal examination of emotional response, cognitive coping and physical recovery among athletes undergoing anterior cruciate ligament reconstructive surgery [dissertation]. Minneapolis: University of Minnesota, 1997.

9. Brena SF, Chapman SL, editors. Management of patients with chronic pain. New York: SP Medical \& Scientific Books, 1983. 
10. Fernandez E, Turk DC. The utility of cognitive coping strategies for altering pain perception: a meta-analysis. Pain 1989;38:123-35.

11. Syrjala KL, Donaldson GW, Davis MW, Kippes ME, Carr JE. Relaxation and imagery and cognitive-behavioral training reduce pain during cancer treatment: a controlled clinical trial. Pain 1995;63:189-98.

12. Janssen SA, Arntz A. Anxiety and pain: attentional and endorphinergic influences. Pain 1996;66:145-50.

13. Janssen SA, Arntz A, Bouts S. Anxiety and pain: epinephrine-induced hyperalgesia and attentional influences. Pain 1998;76:309-16.

14. Williams SL, Kinney PJ. Performance and nonperformance strategies for coping with acute pain: the role of perceived self-efficacy, expected outcomes, and attention. Cognitive Therapy and Research 1991;15:1-19.

15. Whitmarsh BG, Alderman RB. Role of psychological skills training in increasing athletic pain tolerance. The Sport Psychologist 1993;7:388-99.

16. Pen LJ, Fisher CA, Sforzo GA, McManis BG. Cognitive strategies and pain tolerance in subjects with muscle soreness. Journal of Sport Rehabilitation 1995;4:181-94.

17. Schmitz U, Saile H, Nilges P. Coping with chronic pain: flexible goal adjustment as an interactive buffer against pain-related distress. Pain 1996;67:41-51.

18. Haythornthwaite JA, Menefee LJ, Clark MR. Pain coping strategies predict perceived control over pain. Pain 1998;77:33-9.

19. Wiese-Bjornstal DM, Smith AM, Shaffer SM, Morrey MA. An integrated model of response to sport injury: psychological and sociological dynamics. Journal of Applied Sport Psychology 1998;10:46-69.

20. Kleinert J, Samulski DM. Pain-Management in Sports - An intercultural view. In: Carlsson BA, Johnsson U, Wetterstrand F, editors. Proceedings of the Sport Psychology in the New Millennium Conference 2000; 2000 May 24-27; Halmstad, Sweden, 2000;230-4.

21. Melzack R. The McGill pain questionnaire. Major properties and scoring methods. Pain 1975;1:277-99.

22. Duncan GH, Bushnell C, Lavigne GJ. Comparison of verbal and visual analogue scales for measuring the intensity and unpleasantness of experimental pain. Pain 1989;37:295-303.

23. Jensen MP, McFarland CA. Increasing the reability and validity of pain intensity measurement in chronic pain patients. Pain 1993;55:195-203.

24. Price DD, Bush FM, Long S, Harkins W. A comparison of pain measurement characteristics of mechanical visual analogue and simple numerical rating scales. Pain 1994;56:217-26.
25. Kleinert J. Zur Erfassung des Schmerzbewaltigungsstatus bei Verletzungen (SBS-V). Unpublished observations, 2000 [cited 2001 april 30]. Disponível em URL: http://www.dshs-koeln.de/psi/unpubl.htm.

26. Keefe FJ, Crisson J, Urban BJ, Williams DA. Analyzing chronic low back pain: the relative contribution of pain coping strategies. Pain 1990; 40:293-301.

27. Spinhoven P, Kuile MMT, Linssen CG, Gazendam B. Pain coping strategies in a Dutch population of chronic low back pain patients. Pain 1989; 37:77-83.

28. Spinhoven P, Linssen CG. Behavioral treatment of chronic low back pain. I. Relation of coping strategy use to outcome. Pain 1991;45:29-34.

29. Keefe FJ, Caldwell DS, Martinez S, Nunley J, Beckham J, Williams DA. Analyzing pain in rheumatoid arthritis patients. Pain coping strategies in patients who have had knee replacement surgery. Pain 1991;46: 153-60.

30. Keefe FJ, Kashkar-Zuck S, Robinson E, Salley A, Beaupre P, Caldwell DS, et al. Pain coping strategies that predict patients' and spouses's ratings of patients' self-efficacy. Pain 1997;73:191-9.

31. Unruh AM. Gender variations in clinical pain experience. Pain 1996; 65:123-67.

32. Kolt GS, Kirkby RJ, Lindner H. Coping processes in competitive gymnasts: gender differences. Percept Mot Skills 1995;81:1139-45.

33. Rosenstiel AK, Keefe FJ. The use of coping strategies in chronic low back pain patients: relationship to patients characteristics and current adjustment. Pain 1983;17:33-44.

34. Holmes JA, Stevenson CAZ. Differential effects of avoidant and attentional coping strategies on adaptation to chronic and recent-onset pain. Health Psychol 1990;9:577-84.

35. McCaul KD, Malott JM. Distraction and coping with pain. Psychol Bull 1984;95:516-33.

36. Brewer BW, Karoly P. Effects of attentional focusing on pain perception. Motivation and Emotion 1989;13:193-203.

37. Miltner W, Jr RJ, Braun C, Larbig W. Somatosensory event-related potentials to painful and non-painful stimuli: effects of attention. Pain 1989; 38:303-12.

38. Botterill C, Flint F, Ievleva L. Psychology of sports injury. In: Zachazewski JE, Magge DJ, Quillen WS, editors. Athletic injuries and rehabilitation. Philadelphia: W.B. Saunders, 1996;791-804.

39. Ievleva L, Orlick T. Mental links to enhanced healing: an exploring study. Sport Psychologist 1991;5:25-40. 